\title{
Development of two-redundant multi-turn absolute encoder based on small modulus reducer
}

\author{
Wei Xiong ${ }^{1,2, *}$, Yajun $\mathrm{Ma}^{1,2}$, Zhen Wang ${ }^{1,2}$, Weiwei $\mathrm{Jiao}^{1,2}$, Wei $\mathrm{Shi}^{1,2}$, Wenzhang $\mathrm{Li}^{1,2}$, and \\ Peng Zhao ${ }^{1}$ \\ ${ }^{1}$ Beijing Institute of Precision Mechatronics and Controls, Beijing, 100076, China \\ ${ }^{2}$ Laboratory of Aerospace Servo Actuation and Transmission, Beijing, 10076, China
}

\begin{abstract}
In order to meet the development needs of aerospace servo technology, the angular displacement sensor, which compact structure, high reliability, and be able to adapt to harsh working environments is used for the measurement and feedback of the high speed of the servo motor output shaft. The design of a two-redundant multi-turn absolute encoder based on a single-turn absolute encoder and a precision small modulus reducer, which realizes the high speed measurement of the servo motor shaft at $8000 \mathrm{rpm}$. Product performance meets the technical indicators of similar products of HEIDENHAIN encoders, can withstand high temperature, long working life, good dynamic performance, small space and light weight. System test and simulation analysis show that the design technical scheme is effective and feasible, can meet the requirements of the servo system.
\end{abstract}

Keywords: Two-redundant, Multi-turn, Encoder.

\section{Introduction}

With the development of modern servo technology, servo system for supporting the use of angular displacement sensors put forward higher requirements: more compact structure, higher reliability, better environmental adaptability, higher speed. In order to be able to adapt to the harsh working environment and limited space structure, the motor requires low-speed precise control and high-speed accurate positioning, which makes the servo technology urgently need a high-performance motor feedback system. The research and application of multi-loop double redundant absolute high speed encoder is to solve the measurement and feedback of modern servo technology. Since absolute encoders are widely used in servo motor speed measurement and closed-loop control of the system, their reliability directly affects the performance and reliability of the servo system. As the servo system has higher and higher requirements for product reliability, in the measurement and feedback of the servo motor speed, the angular displacement sensor has very strict

\footnotetext{
* Corresponding author: xiongwei99@163.com
} 
restrictions on the structural space and weight, and the axial height is required to be no more than $22.5 \mathrm{~mm}$. The space range with a diameter of not more than $50 \mathrm{~mm}$ realizes the total structure, which meets the multi-turn absolute measurement of the encoder spindle input 8000rpm high speed, 14-bit resolution, two redundant design, and the total weight of the product cannot be greater than $60 \mathrm{~g}$. The existing potentiometer is angular linear displacement sensor cannot meet the requirements, so encoder technology research is needed[1]. The newly developed two redundant encoder tests have met the system performance index requirements.

\section{Encoder overall scheme design}

Two redundant absolute encoders are used for angle absolute position measurement and feedback control of servo motors at all levels. The encoder realizes multi-turn absolute Angle measurement and can rely on the mechanical device to remember the zero position information after the power supply is disconnected. The encoder adopts the principle of Hall encoder to realize absolute encoding within a measurement range greater than \pm 64 circles[2,4,6]. On the basis of single-turn magnetic encoder, the absolute encoding of encoder in more than \pm 64 turns is realized through small modulus mechanical gear reducer. In order to improve the reliability requirements of the system, two redundant design schemes are adopted, and the SPI interface is output. The system block diagram is shown in Figure 1.

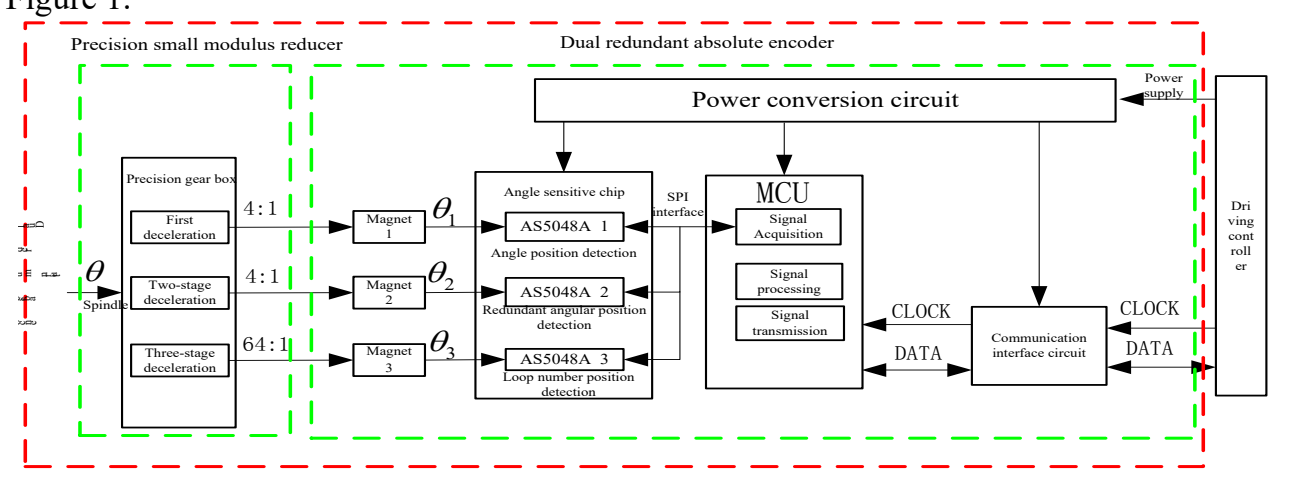

Fig. 1. The block diagram of the dual redundant multi-turn absolute encoder system.

In order to realize the spindle input speed measurement of 8000rpm, the multi-turn encoder adopts the design of its own small modulus reducer, and realizes the multi-stage deceleration through the small modulus reducer. Three AS5048A chips are used to measure the laps and $0-360^{\circ}$ with internal angle measurement and redundant measurement respectively. The output measurement angle of the encoder is composed of the sum of the number of turns and the angle within $0-360^{\circ}$, and the communication transmission is based on the SPI interface mode. The reduction ratio of the primary reduction and the secondary reduction in the precision gearbox is $4: 1$. The first speed reducer is to measure the position of magnet 1 within $0-360^{\circ}$, the second speed reducer is to measure the position of redundant magnet 2 within $0-360^{\circ}$, and the first speed reducer and the second speed reducer are to measure the corresponding position of redundancy. The reduction ratio of the threestage reduction is $64: 1$, and the magnet 3 is installed at the three-stage reducer for lap counting. The final solution angle of the encoder is the synthesis of the reading $\theta 1$ corresponding to AS5048A at the one-stage reducer plus the reading $\theta 3$ corresponding to AS5048A at the three-stage reducer. 


\section{Encoder overall design scheme}

\subsection{System principle}

The encoder uses the magnetic sensitive chip AS5048A made by AMS of Austria. AS5048 is a $360^{\circ}$ angular position sensor with 14-bit high-resolution output. The system can achieve a maximum accuracy of $0.05^{\circ}$ through external microcontroller linearization and averaging [3,5]. The principle of the encoder is that the magnetic field change of the AS5048A sensitive induction magnet is processed by the Sigma Data analogy-to-digital converter and the digital signal processing algorithm inside the AS5048A, and the SPI interface outputs high-precision angle information. The AS5048A control module accesses the internal registers of the AS5048A and AS5048A is configured. After the configuration is successful, the measured angle information and the data representing the magnetic field size are obtained [7]. The principle structure is shown in Figure 2.

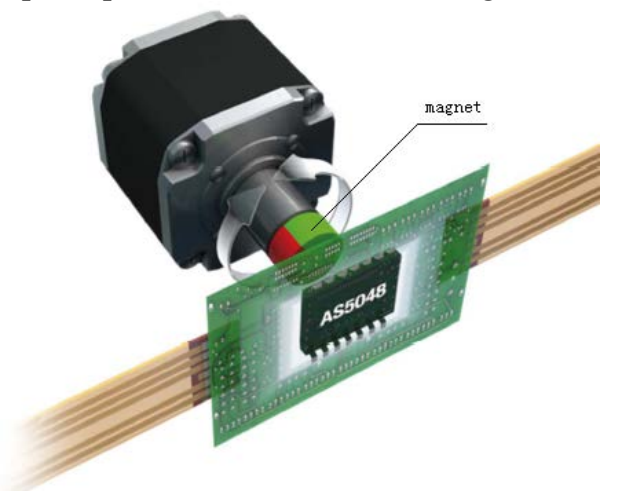

Fig. 2. Principle structure diagram.

The zero position can be programmed via SPI, which simplifies the entire system, because the assembled magnet at the zero position does not need to be mechanically aligned, which facilitates the installation and debugging of the encoder system. The internal structure of AS5048A chip is shown as in Figure 3.

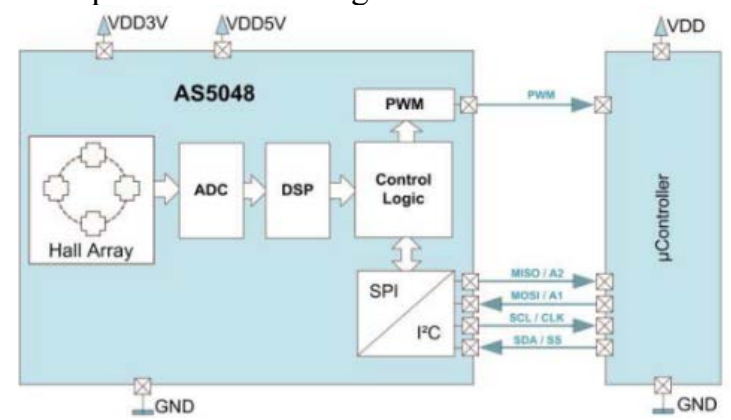

Fig. 3. Internal structure of AS5048A chip.

Working principle of dual-redundant encoder: The AS5048A chip uses the Hall array sensor to detect the change of the magnetic field by perpendicular to the chip surface to solve the corresponding code value to achieve absolute encoding. Figure 4 shows the magnetic field distribution diagram of the magnet, where $\mathrm{X}$ represents the direction parallel to the surface of AS5048A, and Y represents the direction perpendicular to the surface of AS5048A. 

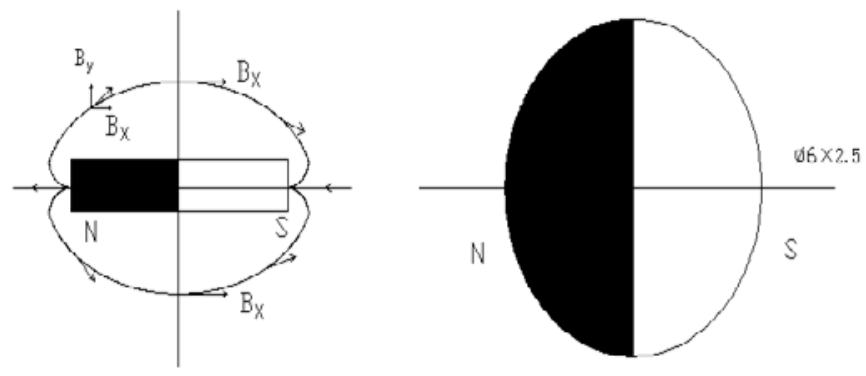

Fig. 4. Magnetic field distribution of magnet.

Figure 5 is a schematic diagram of the AS5048A chip track distribution. As the AS5048A has a 14-bit resolution, it is equally divided in the range of $360^{\circ}$, achieving $214=16384$ code values. The angle corresponding to each code value is:

$$
\frac{360^{\circ}}{16384}=0.0219^{\circ}
$$

The number of code values corresponding to by in the figure is $\mathrm{M}$ and the angle $\theta$.

$$
\theta=\frac{360^{\circ}}{16384} * M
$$

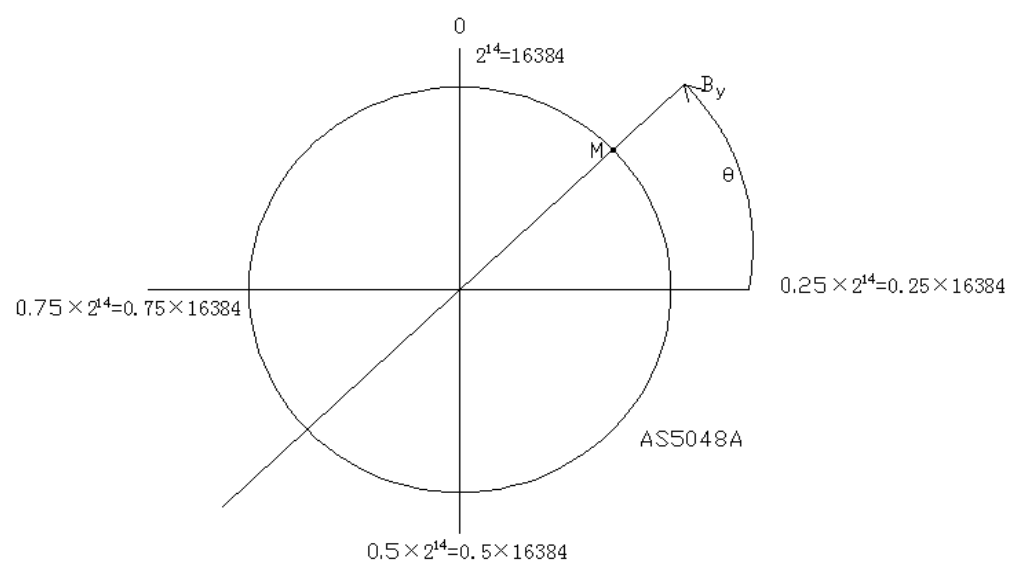

Fig. 5. Schematic diagram of AS5048A chip track distribution.

\subsection{Encoder structure design}

The design principle of two-redundant multi-turn absolute encoder is small size and light weight. The encoder is mainly composed of: shell, hollow shaft, mechanical gear reducer, magnet, screw, circuit system, wire, and the shape structure of the encoder. The size is shown in Figure 6, the external structure size is $\varphi 50 \times 22.5(\mathrm{~mm})$, and the axial height of the reducer is $10.2 \mathrm{~mm}$. 


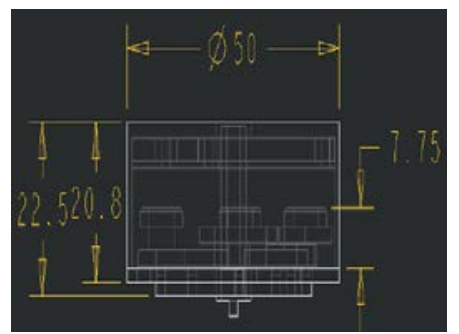

Fig. 6. Three-dimensional structure diagram of the encoder.

The principle of encoder structure design mainly has the following aspects:

- The volume is small, the space design size is $\varphi 50 \times 22.5$, and the mass is not more than $0.06 \mathrm{~kg}$.

- The gear resistance is small, and the gear adopts the non-magnetic material Peek, which contains graphene, which helps self-lubricating, reduces the gear rotation resistance and is non-magnetic, and will not cause the magnetic field around the magnet to change.

- The axial movement of the main shaft is $\pm 1.5 \mathrm{~mm}$ to ensure that the axial movement of the motor shaft will not affect the performance of the reducer.

\subsection{Design of small modulus reducer}

From the preliminary design of the reducer transmission ratio and the preliminary check of strength, it can be found that: (1) When the gear adopts 0.5 module, the minimum gear diameter is $4 \mathrm{~mm}$, the number of teeth is 8 , and the number of teeth is less. The layout of the reducer shaft system should be optimized and the transmission should be increased. The number of teeth of the smallest gear in the shaft system; (2) In the strength check, the maximum tooth surface contact stress is about $0.5 \mathrm{Mpa}$ when the 0.5 module is used in the calculated transmission shaft system, and the maximum tooth root bending stress is about 43.2Mpa, which is 0.3 The maximum tooth surface contact stress at the modulus is about $0.468 \mathrm{Mpa}$, and the maximum bending stress at the tooth root is about $41.8 \mathrm{Mpa}$. There is surplus gear strength. When 0.4 modulus is adopted, the minimum number of gear teeth is 15 and the idler must be removed. The plan has to be changed a lot, See appendix for details; (3) Such as peek with better comprehensive performance, can be used as gear materials to improve the comprehensive performance of gears[8,10]. The following is to reoptimize the design of the subtractor gear transmission by changing the gear modulus to 0.3 .

\subsubsection{Optimized design of the subtractor drive chain}

(1) Constraints

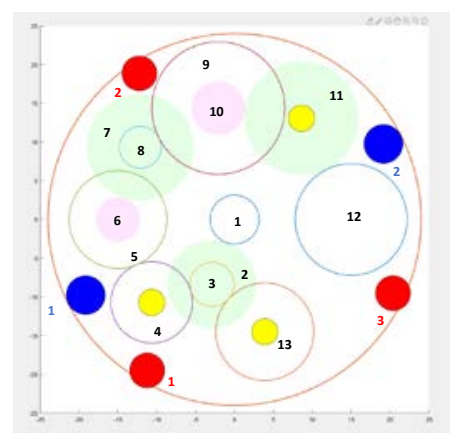

Fig. 7. Reducer drive chain. 
The layout of each gear is shown in Figure 7. The axis of gears 5 (6) and 12 are horizontally symmetrical. The positions of all gear shafts can be moved. First, the number of teeth is optimized, and finally the mounting holes is arranged according to the space $[9,11]$. The entire gear train needs to meet the following conditions:

The transmission ratio should be satisfied the following conditions.

1) Transmission ratio

$$
\left\{\begin{array}{l}
\mathrm{i} 113=4 \\
\mathrm{i} 15=4 \\
\mathrm{i} 112=64
\end{array}\right.
$$

2) Minimum number of teeth

The number of gear teeth must be greater than or equal to 17 , that is

$$
\mathrm{z}_{\mathrm{i}} \geq 18(i=2, \cdots 13)
$$

The number of teeth of 1 input shaft should be the largest, namely

$$
\mathrm{z}_{1}=\max \left(\mathrm{z}_{1}\right)
$$

3) No interference between gears

- The distance between the axis of gears 7, 9, 11 and the center of 1 is greater than the sum of the addendum radius.

$$
l_{1 i}>r_{a i}+r_{a 1}(i=7,9,11)
$$

- The distance between the shaft centers of gears 6 and 7 is greater than the sum of the radius of the addendum circle of 5 and 8 ; the shaft centers of gears 8,9 are greater than the sum of the radius of the addendum circle of gears 9 , and 10 .

$$
\left\{\begin{array}{l}
l_{6,7}>r_{a 5}+r_{a 8} \\
l_{8,9}>r_{a 9}+r_{a 10}
\end{array}\right.
$$

4) The diameter of the shaft cannot be too small

The distance between the shaft centers of the gears 4,11 and 13 and the teeth is greater than the minimum diameter.

$$
\left\{\begin{array}{l}
l_{3,13}>r_{a 12}+r \\
l_{3,4}>r_{a 2}+r \\
l_{10,11}>r_{a 11}+r
\end{array}\right.
$$

5) The number of meshing teeth is relatively prime 


$$
\left\{\begin{array}{l}
\left(z_{1}, z_{2}\right)=1 \\
\left(z_{3}, z_{13}\right)=1 \\
\left(z_{3}, z_{4}\right)=1 \\
\left(z_{4}, z_{5}\right)=1 \\
\left(z_{6}, z_{7}\right)=1 \\
\left(z_{8}, z_{9}\right)=1 \\
\left(z_{10}, z_{11}\right)=1 \\
\left(z_{11}, z_{12}\right)=1
\end{array}\right.
$$

(2) Results of the number of teeth

According to the constraints of the wheel train, the 4 combinations in the table below can be obtained.

Table 1. Gear transmission system tooth number combination.

\begin{tabular}{|c|c|}
\hline Gear & Number of teeth \\
\hline 1 & 21 \\
\hline 2 & 38 \\
\hline 3 & 19 \\
\hline 4 & 35 \\
\hline 5 & 42 \\
\hline 6 & 19 \\
\hline 7 & 46 \\
\hline 8 & 18 \\
\hline 9 & 57 \\
\hline 10 & 23 \\
\hline 11 & 49 \\
\hline 12 & 48 \\
\hline 13 & 42 \\
\hline $\mathrm{i} 113$ & 4 \\
\hline $\mathrm{i} 15$ & 4 \\
\hline $\mathrm{i} 113$ & 64 \\
\hline $\mathrm{m}$ & 0.3 \\
\hline
\end{tabular}

\subsubsection{Finite element simulation analysis of small modulus reducer}

Modal analysis is used to determine the natural frequencies of components and to avoid or minimize excitation on these frequencies, thereby eliminating excessive vibration and noise. For the gear train of the encoder, since it works under no load, the excitation that may exist is the influence of the gear meshing, that is, the gear meshing frequency [12]. The work of modal analysis is to make the modal frequency avoid the meshing frequency of the gear train.

(1) In the simulation analysis, each gear adopts peek material, and the bottom plate adopts aluminium alloy. Through the finite element mesh modelling of the threedimensional model. The gear meshing contact surface is set as frictional contact, the friction coefficient is set as 0.2 , the gear shaft and the base are set as frictionless contact, and the rest of the contact surfaces are set as binding contact [13]. Fix the surface of the 
inner hole of the center shaft of the base plate (assuming it is consolidated with the output shaft of the motor), and use this as a boundary condition to calculate the first six modes of the encoder.

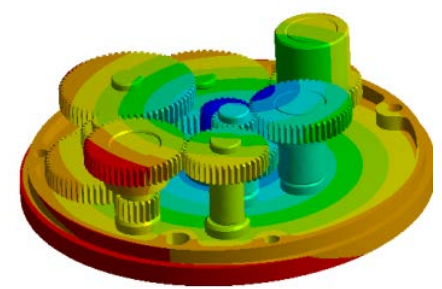

Fig. 8. The first-order mode $2352 \mathrm{~Hz}$.

The first-order resonance frequency of the encoder represents the torsional vibration of the entire encoder along the motor shaft hole, and the torsional mode is difficult to be excited under actual working conditions.

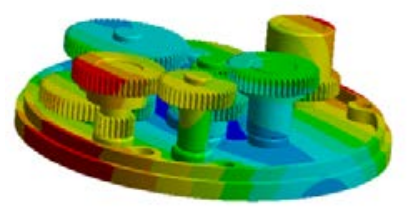

Fig. 9. The second-order mode $2404 \mathrm{~Hz}$.

The second-order resonance of the encoder is represented by the overall oscillation, which is caused by the gear train along with the bottom plate, so it will not affect the meshing between the internal teeth.

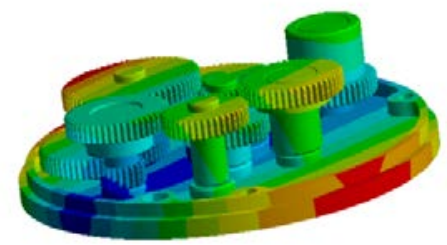

Fig. 10. The third-order mode $2498 \mathrm{~Hz}$.

The third-order and second-order modes belong to the same mode, but the directions are different, because the gear train of the encoder is asymmetric.

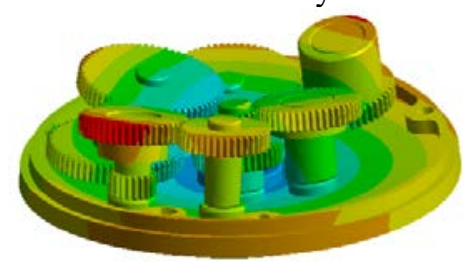

Fig. 11. Fourth-order mode $4752 \mathrm{~Hz}$.

The fourth-order mode shows up and down expansion and contraction vibration of the bottom plate. This mode is more dangerous, because during the bending process of the bottom plate, it is very likely that the teeth will break off and affect the normal working state. 


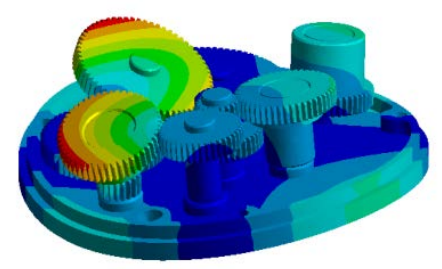

Fig. 12. The fifth-order mode $6120 \mathrm{~Hz}$.

It can be seen from the mode shape and characteristic frequency that the fifth-order mode exhibits the bending of the bottom plate. Like the fourth-order mode, it is very likely to cause the gears to disengage, and it is also a more dangerous mode.

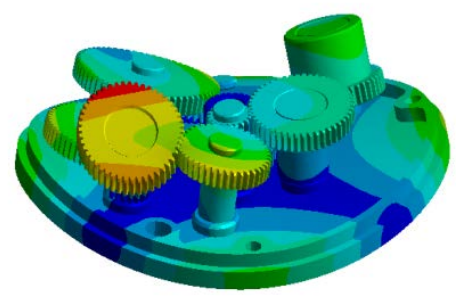

Fig. 13. The sixth-order mode $6581 \mathrm{~Hz}$.

The sixth-order mode is still the bending vibration of the bottom plate, but the direction is different from that of the fifth-order mode.

For an encoder under normal working conditions, the meshing frequency is the product of the rotation frequency and the number of teeth[14]. The meshing frequency of the mutually meshing teeth is the same, so the first tooth is used for calculation: the number of teeth is 24 , the speed is $8000 \mathrm{rad} / \mathrm{m}$, and the meshing frequency:

$$
f=24 \times 8000 / 60=3200 \mathrm{~Hz}
$$

The frequency of rotation is far from the frequency of each mode, so there will be no resonance.

(2) Analysis of the contact stress of the gear at the input of the encoder's precision small modulus reducer.

Since the encoder has no load, there is no load torque. In the simulation of contact stress and subsequent transient analysis, the output torque of the selected motor is used. According to the selected relevant motor, the output torque is $1.3 \mathrm{~mm} * \mathrm{~N}$. Using this torque as the input torque, the contact stress of the first tooth and the second tooth at the input end is analysed, and the contact stress simulation analysis is carried out by modelling the finite element mesh of the first and second gears.

The simulation results show that the maximum stress occurs at the root of the meshing tooth, which is consistent with the actual situation. The maximum stress is $1.6 \mathrm{Mpa}$, and the selected peek can meet the stress limit requirements.

(3) Simulation conclusion analysis

In the simulation analysis, the constraint mode is used, and the actual working state is compared specifically, that is, the encoder is sleeved on the motor output shaft. Therefore, in the simulation analysis, the inner surface of the fixed encoder base plate connected to the motor shaft hole can correspond to the actual work status. Judging from the overall modal analysis results of the encoder, the first few modes are mainly manifested as the vibration of the circuit board, because the circuit board is fixed by three bolts in the design, and this 
support method makes the bending characteristic frequency of the circuit board lower. The vibration of the circuit board itself will not directly affect the working state of the gear train, but the supporting conditions make the bending of the circuit board drive the casing and the bottom plate, and the bending of the bottom plate is very likely to cause the gears to mesh and disengage, thereby affecting the working performance. Although the calculation results show that the fourth-order modal frequency is close to the meshing frequency of the gear at the highest speed, this can be avoided in the subsequent arrangement. The specific measure is to add a support column between the support board and the circuit board, which can change the corresponding circuit board bending frequency, and even eliminate the bending mode of the circuit board completely if necessary.

Regarding the mode of the gear train itself, the lowest-order gear shaft bending mode is much higher than the meshing frequency, so the gear train itself will not resonate and is safe enough. As for the contact stress, since the encoder works under no load, the driving torque of the motor is far from the value that causes the gear to fail, so the safety factor is high enough.

\subsection{Circuit system design}

The multi-turn absolute encoder is mainly composed of power conversion circuit, Angle acquisition circuit, microprocessor circuit and communication interface circuit. The Angle information of AS5048A is collected and stored by the microprocessor circuit through the SPI interface, and the mode instructions of the drive controller are received through the communication interface circuit. After parsing, the Angle, parameters and other information are fed back to the drive controller. The main control part of the encoder selects the smallest system of STM32, which mainly completes the control of signal acquisition, signal processing, and signal transmission. The chip has the characteristics of high performance, low cost, and low power consumption.

The interface circuit chooses MAX3485 RS-485 transceiver to realize the conversion of receiving and sending signals. RE-DE is used in the circuit to control the two-way transmission of data.

\section{Experimental results and analysis}

The dynamic performance test is the most important test for the encoder performance evaluation of the servo system. The dynamic performance test evaluates the overall performance of the encoder. The test data is compared and analysed with the performance characteristics of the similar encoder EQI1131 from HEIDENHAIN. Dynamic test plan: under the complete servo motor test platform, the HEIDENHAIN encoder and selfdeveloped encoder are directly installed at the end of the motor. The whole machine system controller is used to drive the servo motor to drive the encoder to rotate, and the whole machine system tester is used to observe the position characteristic data of the encoder and compare the experimental data of the HEIDENHAIN encoder. The physical composition diagram of the dynamic test system is shown in Figure 16. This diagram is based on the AS5048A encoder test diagram. The HEIDENHAIN encoder test diagram replaces the encoder in the figure with the HEIDENHAIN encoder EQI1131. The position characteristics $(0.02 \mathrm{~Hz}, 10 \mathrm{v})$, transient characteristics $(1.00 \mathrm{~Hz})$, and frequency characteristics of $0.35^{\circ}$ were tested respectively. The test comparison data is shown in the table 3 . 


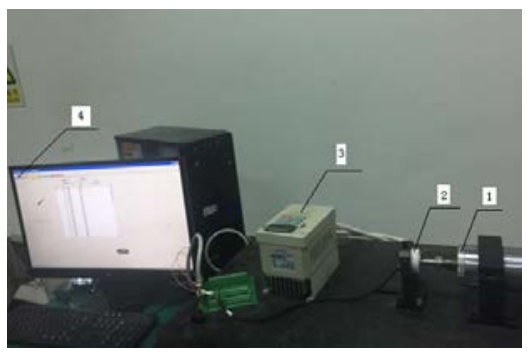

1. Servo motor 2.Encoder 3.Serve motor control 4.Human-computer interaction interface

Fig. 14. The physical composition diagram of the dynamic system.

Table 2. Comparison table of location characteristic data.

\begin{tabular}{|c|c|c|}
\hline Data name & $5048 \mathrm{~A}$ & HEIDENHAIN \\
\hline Maximum forward command $\left({ }^{\circ}\right)$ & 3.844 & 3.976 \\
\hline Maximum negative command $\left({ }^{\circ}\right)$ & -3.901 & -3.942 \\
\hline Maximum forward stroke $\left(^{\circ}\right)$ & 4.277 & 4.273 \\
\hline Maximum negative stroke $\left({ }^{\circ}\right)$ & -4.277 & -4.288 \\
\hline Maximum swing angle $\left({ }^{\circ}\right)$ & 4.277 & 4.281 \\
\hline Loop width iM $\left(^{\circ}\right)$ & 0.082 & 0.076 \\
\hline Zero offset $\left(^{\circ}\right)$ & -0.036 & -0.027 \\
\hline Nominal position gain $\%$ & 1.000 & 1.000 \\
\hline Linearity NL (\%) & 0.500 & 0.690 \\
\hline Position symmetry Ns (\%) & 0.010 & 0.011 \\
\hline
\end{tabular}

Conclusion: comparing the position characteristic data of 5048A encoder and HEIDENHAIN encoder in the table, the difference between AS5048A encoder and HEIDENHAIN data is very small. Among them, the key indicators loop width, linearity, and position symmetry all meet the system index requirements, and the linearity and the position symmetry is better than HEIDENHAIN's similar product EQI1131, and all indicators can meet the requirements of the servo system.

Table 3. Comparison table of transient characteristic data.

\begin{tabular}{|c|c|c|}
\hline Data name & 5048A & HEIDENHAIN \\
\hline Maximum linear speed (\%/s) & 26.957 & 26.977 \\
\hline Overshootop(\%) & 0.000 & 0.000 \\
\hline
\end{tabular}

Conclusion: compare the transient characteristic data of 5048A encoder and HEIDENHAIN encoder in the table. The difference between 5048A encoder and HEIDENHAIN data is small. The maximum linear speed reflects that the encoder has good linear characteristics at maximum speed, and all performance indicators can be satisfied the servo system requirements.

Conclusion: In the comparison table, the frequency characteristics of 5048A encoder and HEIDENHAIN encoder are $0.35^{\circ}$ data, the difference between 5048A and HEIDENHAIN data is small, the amplitude-frequency characteristics data of 5048A encoder has no out-of-tolerance items, and the phase-frequency characteristics have no phase lead or lag. Item, all data indicators can meet the requirements of the servo system.

The system dynamic characteristic test has completed the position characteristic $(0.02 \mathrm{~Hz}, 10 \mathrm{v})$, transient characteristic $(1.00 \mathrm{~Hz})$, frequency characteristic $0.35^{\circ}$ test, which is an important index to quantify the performance of the servo system. In the test, all the 
performance indexes of the 5048A encoder meet the requirements of the servo system, and at the same time reach the performance index of HEIDENHAIN encoder EQI1131.

Table 4. Frequency characteristic $0.35^{\circ}$ of AS048.

\begin{tabular}{|c|c|c|c|c|c|c|c|}
\hline \multicolumn{8}{|c|}{ Frequency characteristic $0.35^{\circ}(1.00 \mathrm{~Hz})(5048: 1$, HEIDENHAIN : 2) } \\
\hline $\begin{array}{c}\text { Freque } \\
\text { ncy } \\
\text { 1f(Hz) }\end{array}$ & $\begin{array}{c}\text { Frequenc } \\
\text { y } 2 \mathrm{f}(\mathrm{Hz})\end{array}$ & $\begin{array}{c}\text { Angular } \\
\text { frequency } \\
1 \mathrm{~L}(w)\end{array}$ & $\begin{array}{c}\text { Angular } \\
\text { frequency } \\
2 \mathbf{L}(w)\end{array}$ & $\begin{array}{l}\text { Amplit } \\
\text { ude } \\
\text { 1L(dB) }\end{array}$ & $\begin{array}{c}\text { Amplitu } \\
\text { de } \\
\text { 2L(dB) }\end{array}$ & $\begin{array}{l}\text { Phase } \\
\text { angle } \\
1 \Phi\left({ }^{\circ}\right)\end{array}$ & $\begin{array}{l}\text { Phase } \\
\text { angle } \\
2 \Phi\left({ }^{\circ}\right)\end{array}$ \\
\hline 0.159 & 0.159 & 1.000 & 1.000 & 0.000 & 0.000 & 0.020 & 0.020 \\
\hline 0.159 & 0.159 & 1.000 & 1.000 & -0.410 & -0.261 & -5.104 & -4.998 \\
\hline 1.592 & 1.592 & 10.000 & 10.000 & -0.512 & -0.413 & -22.621 & -21.987 \\
\hline 3.185 & 3.185 & 20.000 & 20.000 & -1.062 & -1.076 & -40.203 & -38.446 \\
\hline 6.369 & 6.369 & 40.000 & 40.000 & -3.503 & -3.517 & -68.417 & -65.413 \\
\hline 9.554 & 9.554 & 60.000 & 60.000 & -6.019 & -6.101 & -85.485 & -84.118 \\
\hline 12.739 & 12.739 & 80.000 & 80.000 & -8.141 & -8.346 & -100.801 & -99.235 \\
\hline 15.924 & 15.924 & 100.000 & 100.000 & -10.112 & -10.324 & -112.876 & -110.321 \\
\hline 31.847 & 31.847 & 200.000 & 200.000 & -17.622 & -17.719 & -167.747 & -156.686 \\
\hline 47.771 & 47.771 & 300.000 & 300.000 & -22.929 & -23.241 & -227.599 & -205.238 \\
\hline 55.732 & 55.732 & 350.000 & 350.000 & -26.018 & -25.998 & -242.545 & -215.176 \\
\hline
\end{tabular}

\section{Conclusion}

The development of the two-redundant multi-turn absolute encoder of the small modulus reducer is because the traditional potentiometer type angular displacement sensor is difficult to meet the development needs of new aerospace servo models. The improved optimization design based on the single-turn encoder is used for supporting measurement and feedback of servo system. Small modulus reducer, two redundant multi-turn absolute encoders, adopts a small modulus reducer design to achieve a high speed input of $8000 \mathrm{rpm}$ of the main shaft. Through the three-stage deceleration redundancy, position and number of turns measurement functions, it has reached the same level of HEIDENHAIN encoder performance index. The experiment verifies the validity of the design scheme. The ability to meet the reliability and environmental adaptability requirements of the servo system is of great significance to the reliability and completeness of the servo measurement system, and thus has significant social and economic benefits and promotion value.

\section{References}

1. A. Häberli et al., IEEE J. Solid-State Circuits31, 6(1996)

2. K. Nakano, T. Takahashi, and S. Kawahito, IEEE Sensors, 4(2003)

3. S. Kawahito et al., Sens. Mater8, 13(1996)

4. J. Lau, P. K. Ko, and P. C. H. Chan, Sens. Actuators A49,8(1995)

5. A. Nathan, A. M. J. Huiser, and H. P. Baltes, IEEE Trans. Electron Devices32,8(1985)

6. H. P. Baltes and R. S. Popovic, Proc. IEEE74, 26(1986)

7. J. J. Clark, Sens. Actu.ators A24,10(1990)

8. Van de Straete H, Degezelle P, Schutter JD, Belmans RJM. IEEE/ASME Trans Mechatron 3,8(1998)

9. Roos F, Johansson H, Wikander J. Mechatronics 16,10(2006)

10. Cusimano G. Mech Mach Theory42,18(2007) 
11. Giberti H, Cinquemani S, Legnani G. Mechatronics20,7(2010)

12. Verstraten T, Mathijssen G, Furnémont R, Vanderborght B, Lefeber D.Mechatronics30 6(2015)

13. Hassan W, Wang B. Pro-ceedings of the 7th international power electronics and motion control conference, 2. Harbin, China,7(2012)

14. Giberti H, Cinquemani S, Legnani G. Mech Based Des Struct Mach39,17(2011) 